\title{
Stability of low-dimensional multicomponent dilute Bose gases
}

\author{
Alexei K. Kolezhuk* \\ Institut für Theoretische Physik C, RWTH Aachen University, 52056 Aachen, Germany and \\ JARA Jülich-Aachen Research Alliance, Research Centre Jülich GmbH, 52425 Jülich, Germany
}

\begin{abstract}
I show that in low dimensions the interactions in dilute Bose mixtures are strongly renormalized, which leads to a considerable change of stability conditions compared to the mean-field results valid in the high-density regime. Estimates are given for the two-component Bose-Hubbard model and for the ${ }^{87} \mathrm{Rb}-{ }^{41} \mathrm{~K}$ mixture.

PACS numbers: $03.75 . \mathrm{Mn}, 67.85 . \mathrm{Fg}, 67.60 . \mathrm{Bc}, 37.10 . \mathrm{Jk}$
\end{abstract}

\section{INTRODUCTION}

Soon after achieving the Bose-Einstein condensation in ultracold dilute atomic gases [1], there came a surge of interest in degenerate quantum gas mixtures that has been continuing unabated up to the present days. Multicomponent Bose condensates were initially realized [2, 3] by using two different hyperfine states of ${ }^{87} \mathrm{Rb}$, later miscible and immiscible spinor condensates of ${ }^{23} \mathrm{Na}$ were observed [4]. Heteronuclear condensates of ${ }^{41} \mathrm{~K}$ and ${ }^{87} \mathrm{Rb}$ have been realized in a trap [5] and in an optical lattice [6]. Very recently, different groups have used Feshbach resonances to engineer two-component Bose condensates with tunable inter- and intra-species interactions in ${ }^{87} \mathrm{Rb}$ ${ }^{39} \mathrm{~K}$ [7], ${ }^{87} \mathrm{Rb}-{ }^{41} \mathrm{~K}$ [8], and ${ }^{87} \mathrm{Rb}-{ }^{85} \mathrm{Rb}$ [9] mixtures.

The interest to multicomponent Bose systems is driven, particularly, by their rich physics: they provide an opportunity to study various phase transformations ranging from demixing of interpenetrating quantum liquids [10, 11] to transitions between different magnetic states in spinor condensates [12, 13], and to a variety of yet unobserved quantum phase transitions predicted to appear in the presence of an optical lattice [14 18]. Both in traps and optical lattices, the realization of highly anisotropic geometries ("cigars" and "pancakes") is possible [19], which provides an opportunity to study the above phenomena in lower-dimensional systems.

The simplest phase transition in a Bose mixture is the phase separation (demixing) in a two-component system, which occurs if the inter-species repulsion overcomes the intra-species one. Existing theoretical studies of this instability are usually performed at the mean-field level by using coupled Gross-Pitaevskii equations [10, 11]. It is well known that in lower dimensions mean-field arguments might become inapplicable in the low density regime [20]. In a one-dimensional Bose gas, with the decrease of the density, the healing length $\xi$ becomes smaller than the average interparticle distance $d$, thus invalidating the mean-field approach. In two dimensions, the situation is more subtle: although the ratio $\xi / d$ does

\footnotetext{
* On leave from: Institute of Magnetism, National Academy of Sciences and Ministry of Education, 03142 Kiev, Ukraine.
}

not depend on the density and falls below unity only in the case of very tight two-dimensional confinement, in the dilute limit the effective coupling constant becomes strongly energy- and density-dependent [21, 22].

For one-dimensional (1D) multicomponent systems, a study beyond the mean-field approximation, based on the bosonization technique 23], is available [24]. However, the approach of Ref. [24] is constrained by the requirement that the inter-species coupling is small compared to the characteristic bandwidth, which becomes too restrictive for dilute systems and leads to a breakdown of bosonization at very low densities.

The goal of the present work is to provide a stability analysis for dilute multicomponent Bose gases in low dimensions, valid for any coupling strength. It is shown that strong renormalization of coupling constants, typical for low-dimensional systems, can lead to a substantial change in the stability conditions compared to mean-field results, particularly in the case of inequivalent species (heteronuclear mixtures); the mean-field answers restore their validity with the increase of density. The outline of the paper is as follows: in Section III I present the generalization of the renormalization group (RG) approach to the multicomponent case, in Section III I apply this general formalism to the simplest case of two bosonic species with density-density interaction, and Section IV illustrates the case of mutually convertible species on the example of spin-one bosons. Finally, Section $V$ contains the discussion of the possible numerical and physical tests of the theory predictions.

\section{RENORMALIZATION GROUP APPROACH FOR A MULTICOMPONENT DILUTE BOSE GAS}

As a starting point for our analysis, we will choose the theory of multi-species bosonic field with pointlike twobody interactions, that effectively describes a system of atoms with typical momenta much smaller than the inverse characteristic potential range (atom size). Effective field theory in combination with the renormalization group analysis is a convenient tool [25 27] that is widely used [28 30] for the study of the cold atom systems.

Consider the following continuum action describing $N$ species of bosons at zero temperature in $d$ spatial dimen- 
sions, coupled by a general quartic contact interaction:

$$
\begin{aligned}
\mathcal{A}_{N} & =\int d \tau \int d^{d} x\left\{\psi_{\alpha}^{*}\left(\partial_{\tau}-\mu_{\alpha}\right) \psi_{\alpha}+\frac{\left|\nabla \psi_{\alpha}\right|^{2}}{2 m_{\alpha}}+U\right\} \\
U & =\frac{1}{2} \sum_{\alpha \beta \alpha^{\prime} \beta^{\prime}} g_{\alpha \beta, \alpha^{\prime} \beta^{\prime}} \psi_{\alpha}^{*} \psi_{\beta}^{*} \psi_{\alpha^{\prime}} \psi_{\beta^{\prime}}
\end{aligned}
$$

Here $\psi_{\alpha}$ are the fields describing bosonic particles with masses $m_{\alpha}, \alpha=1, \ldots N$, and we have set $\hbar=1$. The interaction matrix for Bose fields satisfies the obvious symmetry conditions $g_{\alpha \beta, \alpha^{\prime} \beta^{\prime}}=g_{\beta \alpha, \alpha^{\prime} \beta^{\prime}}=g_{\alpha \beta, \beta^{\prime} \alpha^{\prime}}=$ $g_{\alpha^{\prime} \beta^{\prime}, \alpha \beta}^{*}$. The action (1) can describe a mixture in a continuum as well as in an optical lattice 24.

When all chemical potentials $\mu_{\alpha}$ vanish, the system is at a critical point. At this special point, the physical picture is considerably simplified [31, 32]: there is no self-energy correction so that the full propagator just coincides with the free one $G_{\alpha \beta}(k, \omega)=\delta_{\alpha \beta} /\left(i \omega-\varepsilon_{k}^{(\alpha)}\right)$, where $\varepsilon_{k}^{(\alpha)}=k^{2} / 2 m_{\alpha}$. This is easy to understand physically, since when all $\mu_{\alpha}=0$, the particle density is just zero. Interaction between the particles is, however, renormalized due to multiple scatterings. The renormalized vertex $\Gamma$, defined at the fixed sum of external momenta $Q$, satisfies the following Bethe-Salpeter equation (illustrated in Fig. 1)

$$
\begin{aligned}
\Gamma_{\alpha \beta, \gamma \delta}(Q) & =g_{\alpha \beta, \gamma \delta}-g_{\alpha \beta, \alpha^{\prime} \beta^{\prime}} f_{\alpha^{\prime} \beta^{\prime}}(Q) \Gamma_{\alpha^{\prime} \beta^{\prime}, \gamma \delta}(Q), \\
f_{\alpha \beta}(Q) & =\int \frac{d^{d} k}{(2 \pi)^{d}}\left[\varepsilon_{Q / 2+k}^{(\alpha)}+\varepsilon_{Q / 2-k}^{(\beta)}\right]^{-1} .
\end{aligned}
$$

The simple ladder form of the above equation is due to the fact that contributions from any diagrams containing closed loops vanish [31].

Above the upper critical dimension $d=2, f_{\alpha \beta}(Q)$ converges at $Q \rightarrow 0$ and (2) simply yields the dressed (observable) interaction matrix. For $d \leq 2$ there is a singularity at $Q \rightarrow 0: f_{\alpha \beta}(Q) \propto 1 / Q$ for $d=1$ and $f_{\alpha \beta}(Q) \propto \ln \left(\Lambda_{0} / Q\right)$ for $d=2$, where $\Lambda_{0}$ is the ultraviolet cutoff (for a system in an optical lattice, the parameter $\Lambda_{0}$ has the physical meaning of a lattice cutoff, and in a continuum it has the sense of an inverse characteristic potential range). Thus for $d \leq 2$ one should look at the RG flow of the running coupling matrix $\Gamma(l)=\Gamma(Q) e^{(2-d) l}$ with the change of the scale $Q \mapsto \Lambda_{0} e^{-l}$. Defining the matrices

$$
\begin{aligned}
& \bar{\Gamma}_{\alpha \beta, \alpha^{\prime} \beta^{\prime}}=2 \Gamma_{\alpha \beta, \alpha^{\prime} \beta^{\prime}}\left(m_{\alpha \beta} m_{\alpha^{\prime} \beta^{\prime}}\right)^{1 / 2}, \\
& F_{\alpha \beta, \alpha^{\prime} \beta^{\prime}}(Q)=\delta_{\alpha \beta, \alpha^{\prime} \beta^{\prime}} R_{d}\left(Q_{\alpha \beta}\right),
\end{aligned}
$$

where $m_{\alpha \beta} \equiv m_{\alpha} m_{\beta} /\left(m_{\alpha}+m_{\beta}\right)$ are the reduced masses, $Q_{\alpha \beta}=2 Q\left(m_{\alpha} m_{\beta}\right)^{1 / 2} /\left(m_{\alpha}+m_{\beta}\right)$, and

$$
R_{d}(q)=\int \frac{d^{d} k}{(2 \pi)^{d}} \frac{1}{k^{2}+q^{2}} \rightarrow\left\{\begin{array}{ll}
\frac{1}{2 q}, & d=1 \\
\frac{1}{2 \pi} \ln \frac{\Lambda_{0}}{q}, & d=2
\end{array},\right.
$$

one can write down the $\mathrm{RG}$ equation as

$$
\frac{d}{d l}\left\{\bar{\Gamma}(1-F \bar{\Gamma})^{-1}\right\}=0
$$

Further, using the identity $A X=X A=X-1$ with $X \equiv(1-A)^{-1}$ and its derivative $d X / d l=X(d A / d l) X$, those equations can be rewritten in the following form:

$$
d \bar{\Gamma} / d l+\bar{\Gamma}(d F / d l) \bar{\Gamma}=0 .
$$

For further simplification, it is convenient to redefine the interaction matrix once again. In one dimension $(d=1)$, one can introduce $\widetilde{\Gamma}=F^{1 / 2} \bar{\Gamma} F^{1 / 2}$; then, since $F$ is a diagonal matrix that for $d=1$ is proportional to $1 / Q$, one obtains $d F / d l=F$ and Eq. (5) can be rewritten as

$$
d \widetilde{\Gamma} / d l=\widetilde{\Gamma}-\widetilde{\Gamma}^{2},
$$

which is familiar from the one-component case [33]; the only difference is that the interaction is now a matrix.

In two dimensions $(d=2)$, it is convenient to define $\widetilde{\Gamma}=\bar{\Gamma} / 2 \pi$; then, using the fact that $d F / d l=1 /(2 \pi)$ for $d=2$, one can reduce (5) to

$$
d \widetilde{\Gamma} / d l=-\widetilde{\Gamma}^{2}
$$

Summarizing the above derivation, we see that the RG equations for the interaction matrix of the lowdimensional multicomponent Bose gas can be cast into the common form

$$
d \widetilde{\Gamma} / d l=(2-d) \widetilde{\Gamma}-\widetilde{\Gamma}^{2}
$$

where

$$
\begin{aligned}
& \widetilde{\Gamma}_{\alpha \beta, \gamma \delta}(l)=\Gamma_{\alpha \beta, \gamma \delta}(l)\left(m_{\alpha} m_{\beta} m_{\gamma} m_{\delta}\right)^{1 / 4} / \Lambda_{0}, \quad d=1 \\
& \widetilde{\Gamma}_{\alpha \beta, \gamma \delta}(l)=\Gamma_{\alpha \beta, \gamma \delta}(l) \sqrt{m_{\alpha \beta} m_{\gamma \delta}} / \pi, \quad d=2 .
\end{aligned}
$$

Since the $\widetilde{\Gamma}$ matrices are symmetric, they can always be diagonalized by an appropriate orthogonal transformation. It is worth noting that at the zero-density critical point the equations (8) are exact to all orders in the interaction $\widetilde{\Gamma}$, similar to the one-component case [33].

The above derivation is nothing but a direct generalization of the well-known RG approach to the onecomponent dilute Bose gas [32, 34 36]. Similar to the one-component case, we will use the RG equations (8), derived at the critical point (zero particle density), to describe the gas of a finite but small density, and to stop the RG flow at some scale $l=l^{*}$ where the system is effectively no more dilute, i.e., where the "running" total density $\rho(l)=\rho_{\text {tot }} e^{d l}$ becomes comparable with $\Lambda_{0}^{d}$. This condition determines the stopping scale $l^{*}$ as

$$
e^{d l^{*}}=C_{d} \Lambda_{0}^{d} / \rho_{\text {tot }},
$$

where $C_{d=1}=\frac{2}{\pi^{2}}$ and $C_{d=2}=\frac{1}{2 \pi}$ can be identified by comparing to the known results for the one-component case [36].

\section{TWO-COMPONENT BOSE MIXTURE}

Let us apply the RG approach outlined in the previous section to the simplest case of two non-convertible 


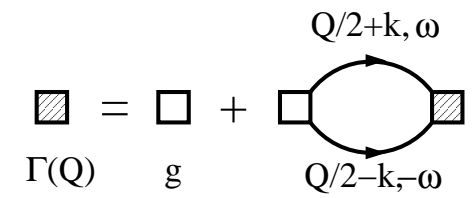

FIG. 1: The renormalized interaction vertex for the multicomponent Bose gas model (11) in the zero density limit, see Eq. (2).

species (i.e., the particle numbers are separtely conserved for each species). The quartic interaction in (1) then takes the form

$$
U\left(\psi_{1}, \psi_{2}\right)=\left(u_{11}\left|\psi_{1}\right|^{4}+u_{22}\left|\psi_{2}\right|^{4}\right) / 2+u_{12}\left|\psi_{1}\right|^{2}\left|\psi_{2}\right|^{2},
$$

so the bare couplings are $g_{11,11}=u_{11}, g_{22,22}=u_{22}$, and $g_{12,12}=u_{12} / 2$. If we denote the entries of the $\widetilde{\Gamma}$ matrix by $\widetilde{u}_{11}$, etc., the RG equations (8) will simplify to

$$
d \widetilde{x} / d l=(2-d) \widetilde{x}-\widetilde{x}^{2},
$$

with $\widetilde{x} \in\left\{\widetilde{u}_{\alpha \beta}\right\}$. We focus on the stability of the mixture. The potential (11) is a quadratic form of the densities, so the necessary condition of the local stability is positiveness of its discriminant:

$$
u_{11}(l) u_{22}(l)-u_{12}^{2}(l)>0,
$$

which must be satisfied by the renormalized interactions $u_{\alpha \beta}(l)$ at all scales up to the RG stopping scale $l=l^{*}$; the intra-species couplings $u_{\alpha \alpha}$ must also be assumed positive to ensure stability. Violation of the condition (13) leads to phase separation (demixing transition) for repulsive interactions between species $\left(u_{12}>0\right)$ and to a collapse in case of attraction $\left(u_{12}<0\right)$. At the level of bare coupling $(l=0)$, Eq. (13) corresponds to the stability condition obtained in the mean-field analysis [10, 11].

\section{A. $d=1$}

In the case of one spatial dimension the solution of $\mathrm{RG}$ equations (12) reads

$$
\begin{aligned}
& \widetilde{u}_{\alpha \beta}(l)=\left\{1+\left(1 / \widetilde{u}_{\alpha \beta}^{(0)}-1\right) e^{-l}\right\}^{-1}, \\
& \tilde{u}_{\alpha \beta}(l)=u_{\alpha \beta}(l) \sqrt{m_{\alpha} m_{\beta}} / \Lambda_{0},
\end{aligned}
$$

where $\widetilde{u}_{\alpha \beta}^{(0)}$ denotes the corresponding bare $(l=0)$ value. A peculiarity of the $1 \mathrm{D}$ case is the tendency of the $\mathrm{RG}$ flow to establish an enhanced symmetry if the interaction between the species is repulsive $\left(u_{12}>0\right)$, even for inequivalent species. Indeed, one can see that at the fixed point $\widetilde{u}_{\alpha \beta}=1$ the potential (11) tends to

$$
U\left(\psi_{1}, \psi_{2}\right) \mapsto \frac{\Lambda_{0}}{2}\left(\frac{\left|\psi_{1}\right|^{2}}{\sqrt{m_{1}}}+\frac{\left|\psi_{2}\right|^{2}}{\sqrt{m_{2}}}\right)^{2},
$$

so the original $U(1) \times U(1)$ symmetry is enhanced to $U(1) \times S U(2)$ at the fixed point.

Let us first consider the repulsive case. Assume that at the microscopic scale $(l=0)$ the mixture is stable, i.e., $u_{12}^{2}<u_{11} u_{22}$. Then the stability condition (13) breaks down starting from a certain scale $l=l_{c}$, determined by

$$
\begin{aligned}
& e^{l_{c}}=1+\frac{\Lambda_{0}\left\{\left[u_{12}^{(m f)}\right]^{2}-u_{12}^{2}\right\}}{u_{12}\left(u_{11} m_{1}+u_{22} m_{2}\right)\left\{u_{12}-u_{12}^{(1 d)}\right\}}, \\
& u_{12}^{(1 d)}=\frac{2 u_{11} u_{22} \sqrt{m_{1} m_{2}}}{u_{11} m_{1}+u_{22} m_{2}}, \quad u_{12}^{(m f)}=\sqrt{u_{11} u_{22}} .
\end{aligned}
$$

The instability develops if $l^{*}>l_{c}$, which translates into the requirement for the density to be low enough,

$$
\rho_{\text {tot }}<C_{1} \Lambda_{0} e^{-l_{c}}
$$

Note that $l_{c}$ is not replacing the RG stopping scale $l^{*}$, but is a new scale that naturally arises in the problem. Here $u_{12}^{(m f)}$ denotes the mean-field result for the instability point [10, 11], but the actual instability, as it is easy to see, generally occurs already at smaller values of $u_{12}$. Indeed, Eq. (15) has a real solution $l_{c}$ if $u_{12}^{(1 d)}<u_{12}<u_{12}^{(m f)}$, and one has $l_{c} \rightarrow \infty(0)$ as $u_{12} \rightarrow u_{12}^{(1 d)}\left(u_{12}^{(m f)}\right)$. If the ultraviolet cutoff $\Lambda_{0}$ is much larger than all the other energy scales, the critical density $\rho_{c}$, below which the instability develops for a given $u_{12}$, becomes cutoff-independent and is given by

$$
\rho_{c}=\frac{C_{1} u_{12}\left(u_{11} m_{1}+u_{22} m_{2}\right)\left\{u_{12}-u_{12}^{(1 d)}\right\}}{\left[u_{12}^{(m f)}\right]^{2}-u_{12}^{2}} .
$$

The above formula can be alternatively viewed as determining the critical value of the interaction $u_{12}^{(c)}$ for a given density $\rho_{\text {tot }}=\rho_{c}$. One can see that the actual instability point $u_{12}^{(c)} \rightarrow u_{12}^{(1 d)}$ if $\rho_{\text {tot }} \rightarrow 0$, and tends to the mean-field answer $u_{12}^{(c)} \mapsto u_{12}^{(m f)}$ when the density becomes large. The resulting phase diagram for the onedimensional case is schematically shown in Fig. 2, For equivalent species $\left(u_{11}=u_{22}, m_{1}=m_{2}\right)$, the points $u_{12}^{(1 d)}$ and $u_{12}^{(m f)}$ coincide, and there is no change in the stability condition compared to the mean-field case.

The case of attractive interspecies interactions $\left(u_{12}<\right.$ $0)$ is much more complicated. The RG flow for $u_{12}(l)$ exhibits a runaway singularity at the characteristic scale $l_{b} \simeq \ln \frac{\Lambda_{0}}{\left|u_{12}\right| \sqrt{m_{1} m_{2}}}$, namely, $u_{12}(l) \rightarrow-\infty$ as $l \rightarrow l_{b}$. It is easy to see that this scale is connected to the presence of molecules (bound states), which exist in one dimension at any strength of the attraction $u_{12}$. Indeed, the energy of the bound state for the contact interaction $U(x)=$ $-\left|u_{12}\right| \delta(x)$ is $E_{b}=-\frac{1}{2} m_{12} u_{12}^{2}$, and the total energy of a molecule with the total momentum $Q$ is $E_{\mathrm{mol}}(Q)=$ $E_{b}+Q^{2} / 2\left(m_{1}+m_{2}\right) ;$ molecules with momenta $|Q|>$ $Q_{b}=\Lambda_{0} e^{-l_{b}}$ can be broken up in collisions, while those with $|Q|<Q_{b}$ are stable.

Molecules represent a new "species", so it is necessary to introduce a new molecular bosonic field $\Psi_{m}$, and in 


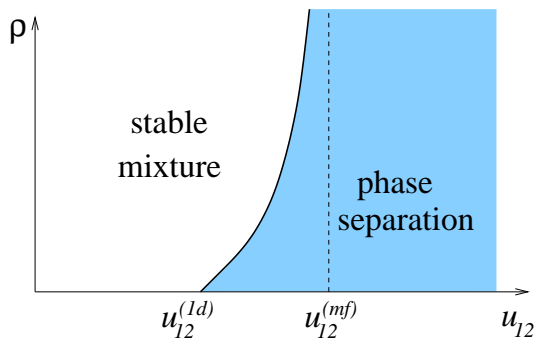

FIG. 2: (Color online). The phase diagram of a twocomponent one-dimensional dilute Bose mixture. The behavior of the critical density $\rho$ in the vicinity of $u_{12}=u_{12}^{(1 d)}$ is linear, cf. Eq. (17).

addition to atom-molecule and molecule-molecule interaction one has to include three-particle amplitudes of the type $\Psi_{m} \psi_{1}^{\dagger} \psi_{2}^{\dagger}$. Presence of those amplitudes invalidates the initial model (11), so the attractive case requires a separate analysis which is beyond the scope of the present paper.

I would like to conclude this subsection with a remark concerning the relation between the present study and that by Cazalilla and Ho 24]. On the basis of the bosonization analysis, they have pointed out that the stability condition is of the mean-field type for large densities (quasicondensate regime) but changes at low densities. Their instability criterion for the low density case (which corresponds to the Tonks limit $m_{\alpha} u_{\alpha \alpha} / \rho_{\alpha} \gg 1$, here $\rho_{\alpha}$ are the densities of individual species) reads

$$
\left|u_{12}\right|>\pi^{2}\left(\rho_{1} \rho_{2} / m_{1} m_{2}\right)^{1 / 2}
$$

and thus is different from that derived in the present work. However, one can argue that the condition (18) should be rather taken as the applicability limit of the bosonization approach itself. Indeed, the perturbative approach of 24] can be expected to break down when the inter-species interaction $u_{12}$ becomes comparable with the characteristic bandwidths $v_{\alpha}=\pi \rho_{\alpha} / m_{\alpha}$ of the individual species. One can also disprove the criterion (18) by considering the limit of hardcore bosons $u_{11}, u_{22} \rightarrow \infty$ on a lattice: in this limit our model is formally equivalent to that of two fermionic species with an on-site interaction, i.e., to the one-dimensional Hubbard model. This model is exactly solvable and from its phase diagram it is known [37] that there is no instability for arbitrary small densities. At the same time, the lower boundary for the instability point $u_{12}^{(1 d)}$, given by (15), diverges as $u_{\alpha \alpha} \rightarrow \infty$, so the absence of instability in the hardcore limit is correctly reproduced in the present approach.

\section{B. $d=2$}

In the two-dimensional case the solutions of RG equations are given by

$$
\widetilde{u}_{\alpha \beta}(l)=\left\{l+1 / \widetilde{u}_{\alpha \beta}^{(0)}\right\}^{-1}, \quad \widetilde{u}_{\alpha \beta}(l)=\frac{m_{\alpha \beta}}{\pi} u_{\alpha \beta}(l) .
$$

In contrast to the one-dimensional case, a tendency to the enhanced $S U(2)$ symmetry is only present for species of equal masses.

Following a similar route as for $d=1$, one obtains the scale $l_{c}$ at which the stability condition (13) breaks down: it is given by the positive root of the equation

$$
\begin{gathered}
\left(\frac{m_{1}-m_{2}}{m_{1}+m_{2}}\right)^{2} l^{2}-\frac{4 \pi^{2}}{m_{1} m_{2}}\left\{\frac{1}{u_{12}^{2}}-\frac{1}{u_{11} u_{22}}\right\} \\
+2 \pi l\left\{\frac{1}{u_{11} m_{1}}+\frac{1}{u_{22} m_{2}}-\frac{4}{u_{12}\left(m_{1}+m_{2}\right)}\right\}=0 .
\end{gathered}
$$

In the general case of unequal masses $\left(m_{1} \neq m_{2}\right)$, the solution $l_{c}$ exists for any $u_{12}$ within the interval $-u_{12}^{(m f)}<$ $u_{12}<u_{12}^{(m f)}$; the scale $l_{c}$ vanishes for $u_{12} \rightarrow \pm u_{12}^{(m f)}$ and diverges at $u_{12} \rightarrow 0$. An instability occurs if $l_{c}<l^{*}$, i.e., if the total density is low enough to satisfy the inequality

$$
\rho_{\text {tot }}<C_{2} \Lambda_{0}^{2} e^{-2 l_{c}} \text {. }
$$

This means that, in contrast to $d=1$, in two dimensions a dilute Bose mixture of two unequal species is always unstable below a certain density, for any value of the interspecies interaction $u_{12}$, see Fig. 3(a). However, for small $u_{12}$ the critical density is exponentially small: according to Eqs. (15), (17), at $u_{12} \rightarrow+0$ the critical value of the density behaves as $\rho_{c} \simeq C_{2} \Lambda_{0}^{2} \exp \left(-A / u_{12}\right)$, where

$$
A=\frac{4 \pi\left(m_{1}+m_{2}\right)}{\sqrt{m_{1} m_{2}}\left(\sqrt{m_{1}}-\sqrt{m_{2}}\right)^{2}} .
$$

At $m_{1} \rightarrow m_{2}$ the coefficient $A$ diverges; actually, in that limit the critical density rapidly goes to zero in the whole interval $0<u_{12}<u_{12}^{(2 d)}$, where

$$
u_{12}^{(2 d)}=\frac{2 u_{11} u_{22}}{u_{11}+u_{22}} .
$$

Thus, the case of equal masses is special: $l_{c}$ exists only for $u_{12}^{(2 d)}<u_{12}^{(m f)}$, resembling the situation in the onedimensional case, see Fig. 3(b). If not only the masses, but also the intraspecies couplings of the two species are equal $\left(u_{11}=u_{22}\right)$, the mean-field answer for the instability threshold is restored at all densities.

The case of attractive interspecies interaction suffers from a similar runaway singularity as in the $1 \mathrm{D}$ case. The scale $l_{b}=\pi\left(m_{1}+m_{2}\right) / m_{1} m_{2}\left|u_{12}\right|$, at which the running coupling $u_{12}(l)$ diverges, is connected to the existence of a bound state (molecule) with the binding energy $E_{b} \propto$ $\exp \left(-2 l_{b}\right)$. Presence of molecules invalidates our original framework (1), so it cannot be directly applied to the attractive case. 

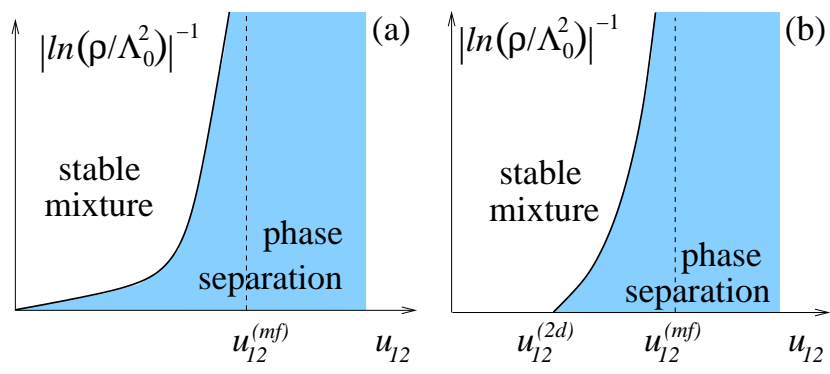

FIG. 3: (Color online) The phase diagram of a two-component dilute Bose mixture in two dimensions: (a) the general case; (b) the special case of equal species masses $m_{1}=m_{2}$. The behavior of the critical values of $1 /\left|\ln \left(\rho / \Lambda_{0}^{2}\right)\right|$ in the vicinity of $u_{12}=0$ and $u_{12}=u_{12}^{(2 d)}$ is linear, cf. Eqs. (20), (21).

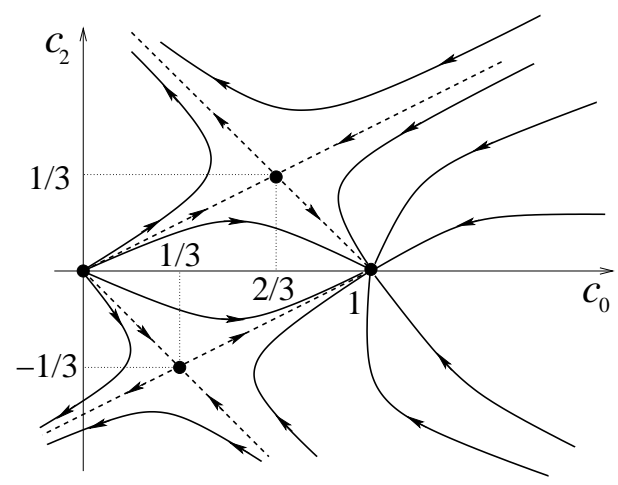

FIG. 4: RG flow diagram for the spinor boson model (24) at zero magnetic field $h=0$, for the one-dimensional case.

\section{IV. $F=1$ SPINOR BOSONS}

In this section I would like to illustrate the application of the presented method to a system with convertible species, where the total particle number of individual species is not conserved. Consider a gas of bosons with the hyperfine spin $F=1$. The contact interaction between two paticles depends on their total spin $S$, so there are two characteristic scattering lengths $a_{S}$ with $S=0$, 2 , and the interaction can be written as [12, 13]

$U=\frac{1}{2}\left\{c_{0} \psi_{a}^{\dagger} \psi_{a^{\prime}}^{\dagger} \psi_{a^{\prime}} \psi_{a}+c_{2} \psi_{a}^{\dagger} \psi_{a^{\prime}}^{\dagger} F_{a b}^{\mu} F_{a^{\prime} b^{\prime}}^{\mu} \psi_{b} \psi_{b^{\prime}}-h\left(S^{z}\right)^{2}\right\}$,

where $a, b \in\{0, \pm 1\}$ denote the three components of the bosonic field, $F^{\mu}$ are the spin-1 matrices, and $S^{\mu}=$ $\psi_{a}^{\dagger} F_{a b}^{\mu} \psi_{b}$ are the spin operators. The couplings are given by $c_{0}=\left(g_{0}+2 g_{2}\right) / 3, c_{2}=\left(g_{2}-g_{0}\right) / 3$, with $g_{S} \propto a_{S}$, and the mass $m$ is the same for all three components. The last term in (24) describes the quadratic Zeeman effect caused by an external magnetic field. This type of interaction favors ferromagnetic spin correlations for $c_{2}<0$, and polar (nematic) ones for $c_{2}>0$ [12, 13].

Although (24) includes just three parameters, the $9 \times 9$ interaction matrix $g_{a b, a^{\prime} b^{\prime}}$ contains generally five different couplings:

$$
\begin{aligned}
g_{++,++}=g_{--,--} & =r \mapsto c_{0}+c_{2}-h \\
2 g_{+0,+0}=2 g_{-0,-0} & =f \mapsto c_{0}+c_{2} \\
2 g_{+-,+-} & =v \mapsto c_{0}-c_{2}+h, \\
g_{00,00} & =u \mapsto c_{0}, \quad g_{+-, 00}=w \mapsto 2 c_{2},
\end{aligned}
$$

where arrows indicate the corresponding bare values.

At zero external field $(h=0)$ the $S U(2)$ symmetry dictates that the effective potential can contain only two constants $c_{0}$ and $c_{2}$, and the RG equations can be cast into the form (12) with $\widetilde{x} \in\left\{\widetilde{c}_{0}+\widetilde{c}_{2}, \widetilde{c}_{0}-2 \widetilde{c}_{2}\right\}$. The "tilded" variables are here defined as $\widetilde{x}=x \mathrm{~m} / \Lambda_{0}$ for $d=1$ and $\widetilde{x}=x m / 2 \pi$ for $d=2$. In one dimension $(d=1)$, there is a nontrivial stable fixed point $\left(\widetilde{c}_{0}=\right.$ $1, \widetilde{c}_{2}=0$ ), which exhibits the enhanced $S U(3)$ symmetry, similar to the double-species case where the fixed point was $S U(2)$-symmetric; the other two fixed points, $\left(\widetilde{c}_{0}=\right.$ $\left.2 / 3, \widetilde{c}_{2}=1 / 3\right)$ and $\left(\widetilde{c}_{0}=1 / 3, \widetilde{c}_{2}=-1 / 3\right)$ are unstable. The corresponding RG flow for $d=1$ is shown in Fig. 4. Both for $d=1$ and $d=2$, there are two runaway flows: the one at $c_{2}>c_{0} / 2$ marks the onset of the pairing state characterized by the formation of bound singlet pairs [3840], while the other runaway flow at $c_{2}<-c_{0}\left(c_{0}>0\right)$ corresponds to the collapse instability when all atoms tend to bind into one giant ferromagnetic "drop". Except of those two runaways, there are no other instabilities.

In presence of the quadratic Zeeman term $(h \neq 0)$ one cannot simply use the full matrix (25) in the RG equations, because a nonzero $h$ leads to a relative shift in chemical potentials of different species, suppressing the $|0\rangle$ states for $h>0$ and $| \pm 1\rangle$ states for $h<0$, respectively. Therefore, if $|h|$ is large enough, one can neglect the suppressed species. For instance, for $h>0$ one comes back to a double-species problem for $|+\rangle$ and $|-\rangle$ states, with a phase separation occuring at $v>r, r>0$, i.e., at $c_{0}+c_{2}>h>c_{2}$. At $c_{2}>0$ this phase separation corresponds to a transition between the nematic and ferromagnetic states. For $h<0$ one effectively obtains a one-component gas, so nothing interesting happens.

\section{SUMMARY AND DISCUSSION}

The examples considered above show that a straightforward generalization of the renormalization group approach for dilute low-dimensional Bose gas [32, 34, 36] to the case of multicomponent systems allows one to obtain nontrivial results concerning the stability of Bose mixtures. For inequivalent species, the stability conditions deviate strongly from the mean-field results; the latter are recovered at higher density.

It is worth noting that the action (1) is quite general: except being applicable to a multicomponent gas in a continuum as well as in an optical lattice, it also arises in quantum magnetism problems dealing with the so-called Bose-Einstein condensation of magnons, induced by a 
strong external magnetic field $H$ in the vicinity of the saturation field $H_{s}$ [41]. In a frustrated magnet the magnon dispersion may have degenerate minima at inequivalent wave vectors, which gives rise to multiple "species" in an effective model [42 44], while $H_{s}-H$ plays the role of the chemical potential.

It would be interesting to test the predictions of the present paper numerically for a one-dimensional twocomponent Bose-Hubbard model with on-site interaction, described by the Hamiltonian

$$
\begin{aligned}
\mathcal{H} & =-\sum_{j, \alpha} t_{\alpha}\left(b_{j, \alpha}^{\dagger} b_{j+1, \alpha}+\text { h.c. }\right) \\
& +\frac{1}{2} \sum_{j, \alpha} U_{\alpha \alpha} \widehat{n}_{j, \alpha}\left(\widehat{n}_{j, \alpha}-1\right)+U_{12} \sum_{j} \widehat{n}_{j, 1} \widehat{n}_{j, 2},
\end{aligned}
$$

where $\alpha=1,2$ labels the two species, $b_{j, \alpha}$ is a bosonic operator at the lattice site $j$, and $\widehat{n}_{j, \alpha}=b_{j, \alpha}^{\dagger} b_{j, \alpha}$ are the corresponding number operators. A simple estimate shows that the necessary requirements can be satisfied at realistic values of the model parameters. Taking, for example, two species with different hopping amplitudes $t_{1}=t, t_{2}=t / 5$ and the same intra-species repulsion $U_{11}=U_{22}=U=3 t$, from (15) one obtains the following window in the inter-species coupling where the phase separation can take place: $0.745<U_{12} / U<1$. Further, fixing, for instance, $U_{12}=0.8 U$, we find from (16) that, in order for the instability to develop, the total density per site $n=\rho_{\text {tot }} / \Lambda_{0}$ must satisfy $n<0.16$, which can be reasonably reached in a density matrix renormalization group calculation [45].

Experimentally, the most promising candidate is the ${ }^{87} \mathrm{Rb}^{-}{ }^{41} \mathrm{~K}$ mixture, for which a very fine control of interactions has been achieved [8]. Assuming that all atoms are in their $|1,1\rangle$ state (as in the setup of Ref. [8]), at low magnetic field $B<100 \mathrm{G}$ the intra-species scattering lengths are approximately given by their $B=0$ values $a_{11} \simeq 97.4$ for ${ }^{87} \mathrm{Rb}\left[46\right.$ ] and $a_{22} \simeq 65.2$ for ${ }^{41} \mathrm{~K}$ [47], in units of the Bohr radius. The effective couplings in a cigar-shaped (quasi-1D) trap with the transverse confining frequency $\omega_{\perp}$ are given by $u_{\alpha \beta}=2 \hbar \omega_{\perp} a_{\alpha \beta}$ [48] (where it is assumed that the characteristic confinement radius $a_{\perp} \gg a_{\alpha \beta}$ ). Thus, from Eq. (15) the critical values for the inter-species scattering length are $a_{12}^{(1 d)} \simeq 68.05$ and $a_{12}^{(m f)} \simeq 79.7$ (independent of $\omega_{\perp}$ ). According to Ref. [8], the dependence of the interspecies scattering length $a_{12}$ on the magnetic field $B$ is very well described by the following expression obtained in Ref. [49]:

$$
a_{12}(B)=a_{b g} \sum_{n=1,2} \Delta_{n} /\left(B-B_{n}\right),
$$

with $a_{b g}=284$ a.u., $\Delta_{1}=37 \mathrm{G}, B_{1}=39.4 \mathrm{G}$, $\Delta_{2}=1.2 \mathrm{G}, B_{2}=78.92 \mathrm{G}$. Therefore, the window for a phase separation $a_{12}^{(1 d)}<a_{12}<a_{12}^{(m f)}$ translates into the field range of 93.8 to $96.3 \mathrm{G}$. Taking $\omega_{\perp}=2 \pi \times 10^{5} \mathrm{~Hz}$, we obtain from Eq. (16) that at the typical particle density $\rho=10^{4} \mathrm{~cm}^{-1}$ the phase separation instability will appear already at $B=B_{c} \simeq 94.9 \mathrm{G}$, i.e., before reaching the mean-field threshold value $B_{m f} \simeq 96.3 \mathrm{G}$. Since recent experiments give hope to the possibility to control the interspecies scattering length $a_{12}$ within a precision better than one Bohr radius [8], a detection of this effect must be within the experimental reach.

A similar estimate can be done for the ${ }^{87} \mathrm{Rb}^{-}{ }^{41} \mathrm{~K}$ mixture in a two-dimensional trap. The couplings in that case 21] are given by $u_{\alpha \beta}=\left(4 \pi \hbar^{3} \omega_{\perp} / m_{\alpha \beta}\right)^{1 / 2} a_{\alpha \beta}$. Using a rough estimate $\Lambda_{0} \sim 1 / r_{\mathrm{Rb}}$, where $a_{\mathrm{Rb}} \simeq 248 \mathrm{pm}$ is the atomic radius of $\mathrm{Rb}$, one can obtain from Eqs. (20), (21) that at the same transverse confining frequency $\omega_{\perp}=2 \pi \times 10^{5} \mathrm{~Hz}$ and a typical density $\rho=10^{5} \mathrm{~cm}^{-2}$ the phase separation instability develops at the critical value of $a_{12} \simeq 54.6$, which with the help of Eq. (27) translates into the critical field $B \simeq 91.4 \mathrm{G}$, well below the mean field value of $96.3 \mathrm{G}$ (and this difference can be increased by lowering the density).

\section{Acknowledgments}

I thank F. Heidrich-Meisner and U. Schollwöck for fruitful discussions. Support by Deutsche Forschungsgemeinschaft (the Heisenberg Program, KO 2335/1-2) is gratefully acknowledged.
[1] See a review in J. R. Anglin and W. Ketterle, Nature (London) 416, 211 (2002).

[2] C. J. Myatt, E. A. Burt, R.W. Ghrist, E. A. Cornell, and C. E. Wieman, Phys. Rev. Lett. 78, 586 (1997).

[3] D. S. Hall, M. R. Matthews, J. R. Ensher, C. E. Wieman, and E. A. Cornell, Phys. Rev. Lett. 81, 1539 (1998).

[4] D. M. Stamper-Kurn, M. R. Andrews, A. P. Chikkatur, S. Inouye, H.-J. Miesner, J. Stenger, and W. Ketterle, Phys. Rev. Lett. 80, 2027 (1998); J. Stenger, S. Inouye, D. M. Stamper-Kurn, H. J. Miesner, A.P. Chikkatur, and W. Ketterle, Nature 396, 345 (1998).

[5] G. Modugno, M. Modugno, F. Riboli, G. Roati, and M. Inguscio, Phys. Rev. Lett. 89, 190404 (2002).
[6] J. Catani, L. De Sarlo, G. Barontini, F. Minardi, and M. Inguscio, Phys. Rev. A 77, 011603(R) (2008).

[7] G. Roati, M. Zaccanti, C. D'Errico, J. Catani, M. Modugno, A. Simoni, M. Inguscio, and G. Modugno, Phys. Rev. Lett. 99, 010403 (2007).

[8] G. Thalhammer, G. Barontini, L. De Sarlo, J. Catani, F. Minardi, and M. Inguscio, Phys. Rev. Lett. 100, 210402 (2008).

[9] S. B. Papp, J. M. Pino, and C. E. Wieman, Phys. Rev. Lett. 101, 040402 (2008).

[10] T.-L. Ho and V. B. Shenoy, Phys. Rev. Lett. 77, 3276 (1996).

[11] E. Timmermans, Phys. Rev. Lett. 81, 5718 (1998). 
[12] T.-L. Ho, Phys. Rev. Lett. 81, 742 (1998).

[13] T. Ohmi and K. Machida, J. Phys. Soc. Jpn. 67, 1822 (1998).

[14] A. Kuklov, N. Prokof'ev, and B. Svistunov, Phys. Rev. Lett. 92, 050402 (2004).

[15] F. Zhou and M. Snoek, Ann. Phys. 308, 692 (2003).

[16] A. Imambekov, M. Lukin, and E. Demler, Phys. Rev. A 68, 063602 (2003).

[17] E. Altman, W. Hofstetter, E. Demler, and M. D. Lukin, New J. Phys. 5, 113 (2003).

[18] A. Isacsson, Min-Chul Cha, K. Sengupta, and S. M. Girvin, Phys. Rev. B 72, 184507 (2005).

[19] For a review see, e.g., I. Bloch, J. Dalibard, and W. Zwerger, Rev. Mod. Phys. 80, 885 (2008).

[20] see, e.g., Chapter 17 of L. Pitaevskii and S. Stringari, Bose-Einstein Condensation (Oxford University Press, 2003)

[21] D. S. Petrov, M. Holzmann, and G. V. Shlyapnikov, Phys. Rev. Lett. 84, 2551 (2000).

[22] M. D. Lee, S. A. Morgan, M. J. Davis, and K. Burnett, Phys. Rev. A 65, 043617 (2002).

[23] F. D. M. Haldane, Phys. Rev. Lett. 47, 1840 (1981).

[24] M. A. Cazalilla and A. F. Ho, Phys. Rev. Lett. 91, 150403 (2003).

[25] J. O. Andersen, Rev. Mod. Phys. 76, 599 (2004).

[26] E. Braaten, M. Kusunoki, and D. Zhang, Ann. Phys. (N.Y.) 323, 1770 (2008).

[27] E. Braaten and H.-W. Hammer, Phys. Reports 428, 259 (2006).

[28] P. Nikolić and S. Sachdev, Phys. Rev. A 75, 033608 (2007).

[29] M. Bijlsma and H. T. C. Stoof, Phys. Rev. A 54, 5085 (1996).

[30] E. Braaten and A. Nieto, Phys. Rev. B 55, 8090 (1997).

[31] D. Uzunov, Phys. Lett. A87, 11 (1981).

[32] M. P. A. Fisher, P. B. Weichman, G. Grinstein, and D. S. Fisher, Phys. Rev. B 40, 546 (1989).
[33] S. Sachdev, Quantum Phase Transitions (Cambridge University Press, 1999).

[34] D. S. Fisher and P. C. Hohenberg, Phys. Rev. B 37, 4936 (1988).

[35] D. R. Nelson and H. S. Seung, Phys. Rev. B 39, 9153 (1989).

[36] E. B. Kolomeisky and J. P. Straley, Phys. Rev. B 46, 11749 (1992).

[37] F. H. L. Essler, H. Frahm, F. Göhmann, A. Klümper, and V. E. Korepin, "The One-Dimensional Hubbard Model" (Cambridge University Press, 2005).

[38] J. Cao, Y. Jiang, and Y. Wang, EPL 79, 30005 (2007).

[39] F. H. L. Essler, G. V. Shlyapnikov, and A. M. Tsvelik, J. Stat. Mech. Theor. Exp., P02027 (2009).

[40] K. Yang, preprint arXiv:0907.4739.

[41] E. G Batyev and L. S. Braginskii, Zh. Eksp. Teor. Fiz. 87, 1361 (1984) [Sov. Phys. JETP 60, 781 (1984)].

[42] T. Nikuni and H. Shiba, J. Phys. Soc. Jpn. 64, 3471 (1995).

[43] G. Jackeli and M. E. Zhitomirsky, Phys. Rev. Lett. 93, 017201 (2004).

[44] A. Kolezhuk and T. Vekua, Phys. Rev. B 72, 094424 (2005).

[45] A. Kleine, C. Kollath, I. P. McCulloch, T. Giamarchi, and U. Schollwöck, Phys. Rev. A 77, 013607 (2008); New J. Phys. 10, 045025 (2008).

[46] E. G. M. van Kempen, S. J. J. M. F. Kokkelmans, D. J. Heinzen, and B. J. Verhaar, Phys. Rev. Lett. 88, 093201 (2002).

[47] S. Falke, H. Knöckel, J. Friebe, M. Riedmann, E. Tiemann, and C. Lisdat, Phys. Rev. A 78, 012503 (2008).

[48] M. Olshanii, Phys. Rev. Lett. 81, 938 (1998).

[49] A. Simoni, M. Zaccanti, C. D'Errico, M. Fattori, G. Roati, M. Inguscio, and G. Modugno, Phys. Rev. A 77, 052705 (2008). 